\title{
Wastewater collection network utilization, using the intelligent technology based on event predictive analysis and GIS
}

\author{
FarhadKatiraei Ph.D. PayamQadirian \\ Master of remote sensing and GIS of Azad university branch of Yazd, GIS department of water and wastewater \\ authority of Isfahan Province \\ F.katiraei@gmail.com \\ Senior Advisor of urban management, Melbourne, Australia \\ pghadirian@gmail.com
}

\begin{abstract}
During the previous years the urban development and growth along with the urban resident increasing, has complicated the planning, designing and utilization of urban facilities. Urban facilities frazzle especially the urban water and wastewater networks and lots of flaws in that network is one of the problems caused the groundwater pollution and serious biometric flaws. Considering the increasing complexity, the classic methods can't satisfied the nowadays urban demands. This article tries to familiar the readers to the novel methods in urban facilities network utilization especially in urban water and wastewater networks. For this aim, at first the initial steps of planning in urban networks will be mentioned and then a spatial system will be introduce based on predictive analysis intelligent technology in utilization management.Designed model in this system is data driven and empirical and has the ability of previous event assessment and the prediction of the future event probability.Also this model is extensible for the other cities and regions that have the wastewater facility and are facing to the accident occurrence in their network.
\end{abstract}

Keywords: Geographic Information System (GIS), urban facilities, Wastewater, Accident, Prediction, Utilization.

\section{Introduction}

During the recent years the urban development and resident growth, has complicated the management of urban networks. On the other hand the technology improvement has provided lots of abilities for the planning, designing and management of urban utilization networks. For example using the different sensors has provided the network situation monitoring in real time procedure. The first step of planning and designing of urban networks, is the spatial data gathering. The classic methods can't satisfy the modern networks demands. The modern technology has given the novel methods such as Lidar and Laser Scanning for the rapid spatial data collection and Grand penetration radar (GPR) for the underground spatial data gathering. GIS projects are performed in urban services for the information providing, designing, engineering and management of urban facilities related to the information. Also the GIS can play the effective role in mechanization of programming and utilization trend of urban facilities.

Qadirian(1376), expressing the way of GIS using in different field, assessed the system usage in urban facilities planning and stated that utilization of this model is inevitable. Jafari et al (1383), evaluated the GIS application in disaster management and water distribution networks. Al-e- Sheikh et al (1388), evaluated the procedures of urban wastewater designing methods, using the GIS. In the international literature some studies has been done in urban wastewater networks such as Crawford (2008) and Rosemary (2008). In this study the way of designing and performance of the model has been used in prediction of urban wastewater network events by the real data of Shahinshar (the north of Isfahan).

\section{Study procedure}

In this study at first the needed data have been gathered, using the documents and library information.Performing the filed study the pipe and manholes have been identified and their location entered into the ArcGIS software (Leipnik M, 1993).Describing information related to the events and accidents have been gathered using the data bank of Shahinshahr water and wastewater authority and was recorded in a data table. Linking the grand database to the wastewater network map, the initial data have been prepared. Making a questionnaire, the weight and importance of effective factors in accident occurrence. This model has been created in GIS medium and has the ability of assessment and prediction of accident occurrence. These events have been evaluated in different pipes with various material, diameter, ages and length.Having the number of annual accidents in consecutive years, the trend of event changes.Generally the accident changes trend in each year has a rising form because of pipe age increasing and fluctuated hydraulic situations (Kettle A, J, 1985). 


\section{Results}

This model application was assessed at first for Shahinshahr, the northern neighbor of Isfahan, and designing the evaluation and prediction of events was provided.

These factors were evaluated and compared, making the questionnaire and using the professors and expert's comments. Considering the previous events and expert comments, the effective factors can be classified in table 1.

Table1. The effective factors on event occurrence in wastewater network

Figure 1 shows the Shahinshahr wastewater networks map, determining the previous event records. The falling events and the events related to the wastewater network obstructionand rejection were separated by the red and yellow colors respectively. The wastewater network events in the study area are divided into two category:

1. The accidents related to the wastewater network falling and corrosion of pipes

2. The accidents related to the wastewater network obstruction and rejection

\section{Fig1. The history of falling and obstruction in the study area}

Based on weights obtained from the AHP method in Expert Choice software, the model is weighted. The weights are assigned to the similar pipes upon to their depth, diameter and material. Thus it can be said that regions nearby to the accident locations obey this situation too. Therefore the previous events are assumed as the case studies (Aramaki T, 2002).

In the table description information the properties of points with accident history. These properties contain the exact address, type of accident, pipe diameter, depth and material, the soil material, the date of accident and the probable accident location. Table 2 shows the results of event evaluation in different spatial location of the study area.

As it can be seen the valves leveling in the northern part of city is an important factor in accident occurrence. In central and western parts of the study area the old age of networks has been determined as an important factor in accident occurrence.

\section{Table2. The final weight assessment results of accidents in Shahinshahr}

Figure 2 shows the history of accidents in northern part of the study area. Also the figure 3 shows the history of accidents in central part of the study area.

\section{Fig2. A schematic of accident history in the northern part of the study area}

\section{Fig3. A schematic of accident history in the central part of the study area}

Using the network analysis in ArcGIS software, the wastewater flow direction is determined in network. Using this process the location of upcoming probable point of wastewater can be guessed. Figure 4 shows the wastewater flow direction in the study area. The black arrows show the flow direction (Esri, 2011).

Fig4. The wastewater flow direction map in the study area.

\section{Result and discussion}

Two important result obtained about the wastewater network pipes relation with accidents.

1. The lesser pipe diameter the higher accident probability. Its reason is the rising of residential complexes and the population increase subsequently.

2. Aging the wastewater pipes and their age rises the events because of the pipe erosion.

Designing the evaluation model and forecasting the urban wastewater network accident, the extension ability of this model to the other regions has been provided. The wastewater event trends was assessed, conjugating the results of GIS and SPSS analysis to each other. in this study the Kendall and Spearman nonparametric tests were done because of the lack of data in pipe diameter and ages.

\section{A. Evaluation of pipe diameter and the events}

In the study area the relation between the pipe diameter and the number of events is shown as equation 1, based on the related data to the event numbers in each pipe diameter:

$$
Y=-0.0275 X+22.441
$$


Wastewater collection network utilization, using the intelligent technology based on event .....

Figure 5 shows the pipe diameter and the number of events relation graph. Upon to the operated statistic tests, based on table 3 it can be understood that the pipe diameter and the number of events have the inverse relation to each other.

\section{Fig5. The pipe diameter and the number of events relation graph}

\section{Table3. The statistical results of the relation between the pipe diameter and event numbers}

\section{B. Evaluation of pipe age and the events}

In the study area the relation between the number of events and the age of pipe is shown as the equation 2 in 20 to 40 years old range. The figure 6 also shows the relation between the pipe age and the number of events.

\section{Fig6. The pipe age and the number of events relation graph}

Table 4 reveals the results of statistical test for the pipe age and event number relation. As it can be seen the pipe age and the event numbers have the direct relation to each other.

\section{Table4. The statistical results of the relation between the pipe age and event numbers}

The effective factors in event occurrence in the urban wastewater network can be divided in two category:

A. The natural factors such as soil type, age of network and etc.

B. The manmade factors such as the objects dropping, industrial wastewater discharge to the pools or the mechanic shovel impact on pipe.

In the present study the effective parameters on wastewater network events listed as below: 1. Performance flaw, 2. the network age, 3. Unacceptable utilization and 4. Mankind factors. In performance flaw the poor material pipe, unsuitable infrastructure, the pipe depth and diameter inappropriateness, unacceptable designing and the soil type had the especial importance. In the study area the soil type and inappropriate infrastructure were determined as the two important factor. Network age was classified in 4 group and the regions with older age and the group with 20-30 years old or more, the events had the more event probability. In unacceptable utilization filed the inappropriate valve levelling, especially in the northern part, has been known as the most important factor in event occurrence. Because the $\mathrm{H}_{2} \mathrm{~S}$ trapping increases the pipe corrosion chance.

The below actions seems essential for the event prevention in the urban facility networks especially the urban wastewater network:

A. Making a regular data bank of event history and the future probable event report.

B. Culture developing in wastewater network usage about the mankind factors affect the events.

C. Making visible the wastewater valves after the asphalting, preventing the corrosion gases aggregation.

D. Using the sensors and the mobile and immobile cameras and the leak detector in the urban wastewater networks.

E. Reasonable infrastructure making at the wastewater pipe performance.

F. Suitable designing of wastewater network pipe with acceptable depth and diameter.

G. Wastewater network modification in the study area with old and worn-out network.

H. Using the high quality pipes such as PE and earthen pipes.

\section{References:}

[1]. Qadirian P. 1375. "GIS application in the urban networks", water and wastewater journal, 33:27-36 (in Persian).

[2]. Jafari H. 1388. "The model of water distribution event management using the GIS", water and wastewater journal, 2:2-16 (in Persian).

[3]. Al-E- Sheikh E. 1388." Wastewater network designing, using the ArcGIS and the calculation software", Environment Science and Technology, issue 11, volume 1, (in Persian).

[4]. Dawie Crawford.2008." implementing a utility Geographic information system for water sewer and electric:Case study of city of Cahoun,Georgia”.pp.14-16.

[5]. Rosemary H.2011.GISP Jordan,"Using GIS to increase the value of a Sanitarysewer preventative Maintenance and inspection program", PP.3-5.

[6]. Leipnik M, Lociga H.1993."Implementation of GIS for water resources planning and management", PP.6-7

[7]. Kettle A.j, Goultor.1985." An analysis of pipe breakage in urban water distribution networks", Can.J.Civ.Eng.PP.286-293

[8]. AramakiT,HanakiK. 2002. "Evaluation of appropriate system for reclaines wastewater in each area of Tokio using GIS based water balance model", -University of Rhode island cooperative Wastewater planning ,PP.2,3

[9]. Esri.2011.Gis data quality best practices for water, wastewater and stormwater utilities, PP.3-4 